\title{
The coiled-coil protein-binding motif in Fusarium verticillioides Fsr1 is essential for maize stalk rot virulence
}

\author{
Yoshimi Yamamurat and Won-Bo Shim
}

Correspondence

Won-Bo Shim

wbshim@tamu.edu

Received 15 January 2008

Revised 17 March 2008

Accepted 27 March 2008
Department of Plant Pathology and Microbiology, Program for the Biology of Filamentous Fungi, Texas A\&M University, College Station, TX 77843-2132, USA

Fusarium verticillioides (Sacc.) Nirenberg (teleomorph Gibberella moniliformis Wineland) is one of the key pathogens of maize stalk rot disease. However, a clear understanding of stalk rot pathogenesis is still lacking. Previously, we identified the F. verticillioides FSR1 gene, which plays a key role in fungal virulence and sexual mating. The predicted Fsr1 protein contains multiple protein-binding domains, namely a caveolin-binding domain, a coiled-coil structure, and a calmodulin-binding motif at the $\mathrm{N}$ terminus and a WD40 repeat domain at the $\mathrm{C}$ terminus. Fsr1 shares significant similarity to a family of striatin proteins that play a critical role in cellular mechanisms that regulate a variety of developmental processes. Significantly, FSR1 function is conserved in Fusarium graminearum, where it also plays a direct role in pathogenesis. In this study, our goal was to determine the motif(s) in Fsr1 that are directly associated with fungal virulence. We complemented the FSR1 knockout $\left(\Delta f_{s} 1\right)$ strain with mutated versions of the FSR1 gene, and determined that the Fsr1 C-terminal WD40 repeat domain is dispensable for vegetative growth and maize stalk rot virulence. We also examined the potential link between FSR1-mediated virulence and cell wall-degrading enzyme ( $\alpha$-amylase, pectinase and cellulase) activities. Further characterization of the $\mathrm{N}$-terminal region revealed that the coiled-coil structure is essential for virulence in F. verticillioides. The coiled-coil domain is involved in a variety of protein-protein interactions in eukaryotic systems, and thus we hypothesize that the interaction between Fsr1 and the putative Fsr1-binding protein triggers downstream gene signalling that is associated with $F$. verticillioides virulence.

\section{INTRODUCTION}

Fusarium verticillioides (Sacc.) Nirenberg (teleomorph Gibberella moniliformis Wineland) is an aggressive pathogen of maize, causing stalk rot and ear rot (White, 1999). The fungus is also known to produce a group of carcinogenic mycotoxins called fumonisins in infested ears (Desjardins et al., 2002). While ear rot research has received considerable attention due to the impact of fumonisins in food and feeds, stalk rot research has lapsed due to the lack of an adequate model system to study the mechanism of virulence in stalk rot-causing fungi. Recently, our group has isolated and characterized a gene in F. verticillioides, designated FSR1, that is critical for maize stalk rot virulence and fertility (Shim et al., 2006). Significantly, disruption of the FSR1 orthologue in Fusarium graminearum, FgFSR1, results in loss of virulence on barley and maize, suggesting that the FSR1-mediated

tPresent address: Laboratory of Plant Resource Sciences, Graduate School of Medicine and Pharmaceutical Science for Research, University of Toyama, 2630 Sugitani, Toyama 930-0194, Japan.

Abbreviation: CWDE, cell wall-degrading enzyme. virulence mechanism is conserved in $F$. graminearum (Shim et al., 2006). FSR1 is predicted to encode a multimodular protein, Fsr1, with four functional domains that are known to be involved in protein-protein interactions. The Fsr1 protein shares significant similarity to a family of animal proteins, e.g. striatin and zinedin, that are critical for a variety of developmental processes and cellular mechanisms. The Fsr1-mediated virulence in $F$. verticillioides provides a new avenue to explore a novel signalling mechanism that controls virulence mechanisms in maize stalk rot pathogens (Shim et al., 2006).

The four putative functional domains in Fsrl include a caveolin-binding domain, a coiled-coil structure, and a calmodulin-binding domain at the $\mathrm{N}$ terminus and a WD40 repeat domain at the $\mathrm{C}$ terminus (Fig. 1a). From the studies performed in mammalian and Drosophila systems (Castets et al., 1996; Moreno et al., 2000), these functional domains are suggested to play critical roles in signalling pathways by mediating protein-protein interactions. The mammalian homologues striatin and zinedin are suggested to be involved in $\mathrm{Ca}^{2+}$-dependent signalling in cells of the central nervous system, and function as scaffolding proteins linking 


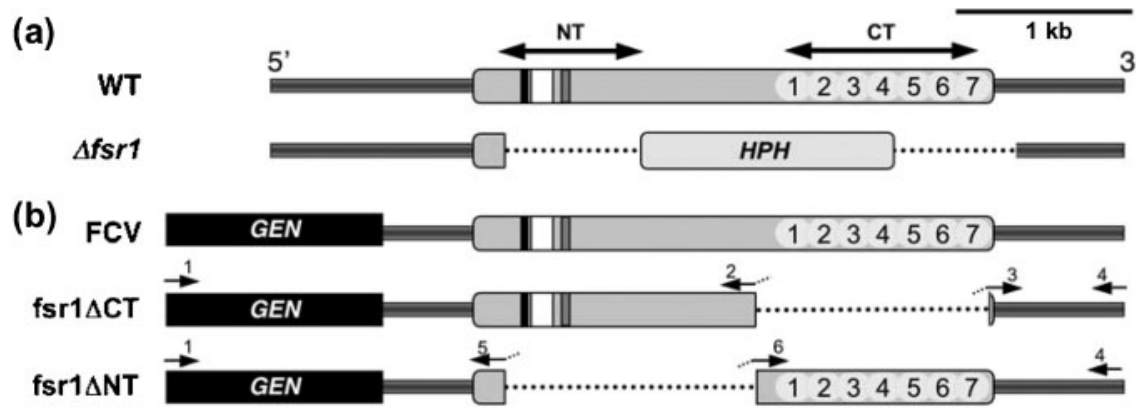

(c)

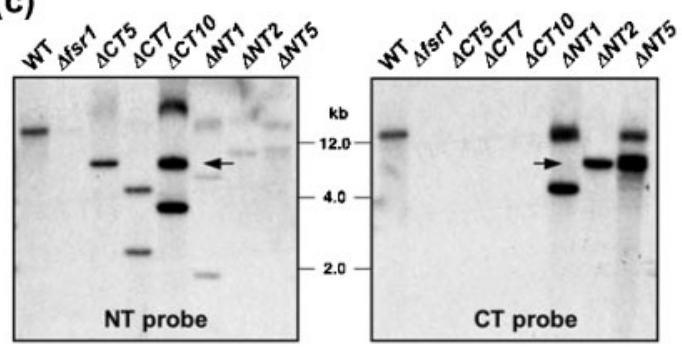

(d)

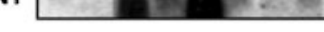

CT

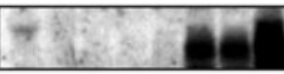

rRNA

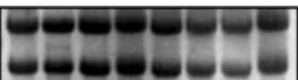

\begin{abstract}
Fig. 1. F. verticillioides FSR1 locus molecular manipulation. (a) Schematic diagram of the FSR1 locus in F. verticillioides wildtype (WT) and $\Delta f s r 1$ strains. The nucleotide sequences that correspond to the caveolin-binding domain, coiled-coil structure, calmodulin-binding motif and WD40 repeat motif are represented by black, white and shaded boxes, and shaded/numbered ovals, respectively. The dotted line indicates a deleted gene region. Double arrows (NT probe and CT probe) show the gene regions used as the probes for Southern and Northern analyses. HPH, hygromycin phosphotransferase gene. (b) The FSR1complementation vector (FCV) (Shim et al., 2006) was used as a template to construct fsr $1 \Delta \mathrm{NT}$ and fsr $1 \Delta \mathrm{CT}$, and fsr $1 \Delta \mathrm{NT}$ and fsr $1 \Delta \mathrm{CT}$ illustrate complementation constructs used to test the functional roles of the $\mathrm{C}$-terminus and $\mathrm{N}$-terminus regions, respectively, in F. verticillioides growth and stalk rot virulence. GEN, geneticin-resistance gene. Arrows depict the primers used to amplify the specific DNA fragments: $1=\mathrm{M} 13 \mathrm{~F}, 2=\mathrm{b} 4-\mathrm{RV}, 3=\mathrm{a}-\mathrm{FW}, 4=\mathrm{FSR} 1-\mathrm{U} 4,5=\mathrm{d}-\mathrm{RV} 2$, and 6=WD40-FW. (c) Southern blot analysis of the selected transformed strains. Fungal genomic DNA was digested with BamHI, and the blot was hybridized with ${ }^{32} \mathrm{P}$-labelled DNA probes for NT and CT (shown in Fig. 1a). The position of molecular size markers (kb) is indicated between the panels. Anticipated bands for fsr $1 \Delta \mathrm{CT}$ and fsr1 $\mathrm{N}$ T constructs when hybridized with the NT and CT probes, respectively, are indicated with arrows. (d) Northern blot analysis of the transformants probed with NT (upper panel) and CT (middle panel) ${ }^{32} \mathrm{P}$-labelled DNA fragments to determine the expression of the fsr $1 \Delta \mathrm{CT}$ and fsr1 $1 \Delta \mathrm{NT}$ constructs, respectively. An ethidium bromide-stained agarose gel (lower panel) confirms uniformity of loading (rRNA) in each lane.
\end{abstract}

signalling and eukaryotic endocytosis (Castets et al., 1996; Moreno et al., 2000). While homologues of Fsr1 can be found in filamentous fungi, their functional role is not fully understood. Prol1 in the homothallic ascomycete Sordaria macrospora was the first striatin orthologue identified and characterized in filamentous fungi. Prol1 plays a critical role in cell differentiation, particularly fruiting body development (Pöggeler \& Kück, 2004). A mutation in the pro11 gene results in loss of fertility and increased aerial hyphae, giving the fungus a cotton balllike appearance. Complementation with a full-length version of the PRO11 gene completely restores the wildtype phenotype. Interestingly, a truncated version containing the coding region for the $\mathrm{N}$-terminal part results only in partial complementation; the colony morphology is not restored, although the formation of fertile fruiting bodies is restored, although at a reduced frequency. These results led the investigators to conclude that while the $\mathrm{N}$ terminus of Pro11 is sufficient to complement fertility, the complete gene is necessary for full function. Pöggeler \&
Kück (2004) concluded that the C-terminal region of Pro11, which contains WD40 repeats, is essential for fungal differentiation and protoperithecia development in S. macrospora. However, the role of these motifs in Fsr1 function associated with $F$. verticillioides development and virulence is not clearly understood.

The Fsr1 protein harbours four putative protein-binding motifs that are known to mediate protein-protein interactions. In particular, a caveolin-binding motif, a coiledcoil structure and a WD40 repeat motif share significant amino acid identity to corresponding motifs in other welldefined animal and fungal homologues (Shim et al., 2006). The Fsr 1 calmodulin-binding motif shares low similarity to striatin and Pro11. Our hypothesis is that one of these four motifs is directly responsible for $F$. verticillioides maize stalk rot virulence. Here, we test our hypothesis through a series of motif-deletion studies and arrive at the conclusion that the coiled-coil motif is critical for Fsr1-mediated virulence in F. verticillioides. We also investigate the roles of these 
putative motifs in the expression of selected cell walldegrading enzymes (CWDEs) in F. verticillioides.

\section{METHODS}

Fungal strains, media and culture conditions. F. verticillioides 7600 (M3125; Fungal Genetics Stock Center) was used as the wild-type strain in this study. F. verticillioides 7598 (Fungal Genetic Stock Center) was used as a female wild-type strain in sexual crosses. The FSR1 disruption-mutant strain $\Delta f_{s} r 1$ has been described previously (Shim et al., 2006). The fungal strains were grown on V8 agar (Shim \& Woloshuk, 2001) at $25{ }^{\circ} \mathrm{C}$ for inoculum preparation and routine maintenance. Colony growth rate, morphology and pigment production were compared on potato dextrose agar (PDA; Difco). To study expression of genes that encode CWDEs, we prepared 'crude stalk juice' by pulverizing 8 -week-old maize stalks in a blender, adjusting the final concentration to $10 \%(\mathrm{w} / \mathrm{v})$ stalk in water, followed by autoclaving. Fungal conidia produced on 7-day-old V8 agar plates were collected

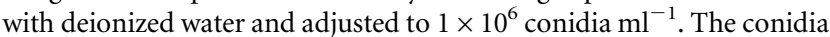
suspension $(1 \mathrm{ml})$ was inoculated into $100 \mathrm{ml}$ crude stalk juice broth and incubated on a rotary shaker $(150$ r.p.m. $)$ at $25{ }^{\circ} \mathrm{C}$ for 7 days.

Generation of Fsr1 motif-deletion mutants. To investigate the functional role of Fsr1 $\mathrm{N}$-terminus and $\mathrm{C}$-terminus regions, we first assembled two complementation constructs, one with a complete deletion of the $\mathrm{N}$ terminus (fsr $1 \Delta \mathrm{NT}$ ) and the other with a complete deletion of the $\mathrm{C}$ terminus (fsr $1 \Delta \mathrm{CT}$ ), and introduced each to the $\Delta f_{s} r 1$ protoplasts (Fig. 1). Both constructs contained the native promoter of FSR1 and were fused to a geneticin-resistance (GEN) marker (Flaherty et al., 2003). FSR1-complementation vector (FCV) (Shim et al., 2006) was used as a template to construct fsr $1 \Delta \mathrm{NT}$ and fsr $1 \Delta \mathrm{CT}$. A doublejoint PCR strategy was used to generate all complementation constructs (Shim et al., 2006; Yu et al., 2004). All primers used in this study are listed in Table 1. Primers used for construction of fsr $1 \Delta \mathrm{NT}$ and fsr $1 \Delta \mathrm{CT}$ are as follows: for the $5^{\prime}$ flanking region of fsr $1 \Delta \mathrm{CT}, \mathrm{M} 13 \mathrm{~F}$ and b4-RV; for the $3^{\prime}$ flanking region of fsr1 $\Delta \mathrm{CT}$, a-FW and FSR1-U4; for the $5^{\prime}$ flanking region of fsr $1 \Delta \mathrm{NT}, \mathrm{M} 13 \mathrm{~F}$ and d-RV2; and for the $3^{\prime}$ flanking region of fsr1 $\Delta$ NT, WD40-FW and FSR1-U4. Subsequently, fsr $1 \Delta \mathrm{CT} \Delta \mathrm{Cav}$ and fsr $1 \Delta \mathrm{CT} \Delta \mathrm{CC}$ complementation constructs were created by amplifying DNA fragments using fsr $1 \Delta \mathrm{CT}$ construct as the template. For construction of fsr $1 \Delta \mathrm{CT} \Delta \mathrm{Cav}$ and fsr $1 \Delta \mathrm{CT} \Delta \mathrm{CC}$, the following primers were used: for the $5^{\prime}$ flanking region of fsr $1 \Delta \mathrm{CT} \Delta \mathrm{Cav}, \mathrm{M} 13 \mathrm{~F}$ and $\mathrm{h}-\mathrm{RV}$; for the $3^{\prime}$ flanking region of fsr1 $\Delta \mathrm{CT} \Delta \mathrm{Cav}, \mathrm{g}-\mathrm{FW}$ and FVLM2F1; for the $5^{\prime}$ flanking region of fsr $1 \Delta \mathrm{CT} \Delta \mathrm{CC}, \mathrm{M} 13 \mathrm{~F}$ and $\mathrm{j}-\mathrm{RV}$; and for the $3^{\prime}$ flanking region of fsr $1 \Delta \mathrm{CT} \Delta \mathrm{CC}$, i-FW and FVLM2F1. The final (nested) PCR round was performed using the primers GeneF and FVLM2F1. The final PCR product sizes of fsr $1 \Delta \mathrm{NT}$, fsr $1 \Delta \mathrm{CT}$, fsr $1 \Delta \mathrm{CT} \Delta \mathrm{Cav}$ and fsr $1 \Delta \mathrm{CT} \Delta \mathrm{CC}$ were 5.1, 4.8, 3.9 and $3.8 \mathrm{~kb}$, respectively. The PCR products were used directly for fungal transformation. All PCR amplifications associated with complementation constructs were performed with the Expand Long Template PCR system (Roche) following the manufacturer's suggested protocol. Fusarium transformations were performed as described previously (Shim et al., 2006). Briefly, protoplasts were prepared and the transformation construct $(10 \mu \mathrm{g})$, fsr1 $1 \Delta \mathrm{NT}$, fsr $1 \Delta \mathrm{CT}$, fsr $1 \Delta \mathrm{CT} \Delta \mathrm{Cav}$ or fsr $1 \Delta \mathrm{CT} \Delta \mathrm{CC}$, was added to the protoplasts $(100 \mu \mathrm{l})$. Transformants were regenerated in Fusarium regeneration agar medium [1 M sucrose, $0.02 \%(\mathrm{w} / \mathrm{v})$ yeast extract (Difco), $1 \%(\mathrm{w} / \mathrm{v})$ agar] containing hygromycin $\mathrm{B}\left(100 \mu \mathrm{g} \mathrm{ml}{ }^{-1}\right.$; Calbiochem $)$ and

Table 1. Primers used in this study

The underlined sequences were added to the $5^{\prime}$ ends of the primers to facilitate fusion PCR.

\begin{tabular}{|ll|}
\hline Primer & \multicolumn{1}{c|}{ Sequence $\left(\mathbf{5}^{\prime} \mathbf{- 3}^{\prime} \mathbf{)}\right.$} \\
\hline M13F & TTGTAAAACGACGGCCAGTGA \\
GeneF & TGAACAAGATGGATTGCACGCAGG \\
b4-RV & CAAAAAGCTGAGAGTGGTAATTCTAATGGAGATAGATTCTTTAC \\
WD40-FW & GTAAAGAATCTATCTCCATTAACCTCGGAGTCCAAATCTGAGCTT \\
d-RV2 & AAGCTCAGATTTGGACTCCGAGGTCACCTC \\
FSR1-U4 & CCTCGGAGTCCAAATCTGAGCTTTCGCATGACGCCCTGGAGGGTG \\
FVLM2F1 & AGGATAACAGCAGTAGATGGCAGC \\
g-FW & AAATGCCGGGTATCGACGAGTAGT \\
h-RV & ACCCTCCAGGGCGTCATGCGACGCGATAGGAACTCGTGGGAGATT \\
i-FW & AATCTCCCACGAGTTCCTATCGCGTCGCATGACGCCCTGGAGGGT \\
j-RV & CATGAGCGCGATAGGAACTCGACGGCTCAGCTTCAGGCTCGGGAG \\
FSR1-U3 & CTCCCGAGCCTGAAGCTGAGCCGTCGAGTTCCTATCGCGCTCATG \\
FSR1-D2 & GTGACTCGTCACTTCTTGCGTGC \\
FSR1-U1 & AATGGGACCTATGATGCAGGGCAA \\
CoilBD-FW & GCACGCAAGAAGTGACGAGTCAC \\
CoilBD-RV & TGGGAGATTGAGAAGCAGGAG \\
FSR-1186-FW & GCTCCTTAACCTTTCGCTCCAG \\
FSR-BD-RV & CTACCTTGGAGGATATGCCAGTG \\
Fv-amylase-F & GAAATTACCACTCTCAGCTTTTTG \\
Fv-amylase-R & GTATCCGCATCGATACAGTCAA \\
FvPG-F & CCCAAGTCTTGTTGTTCTTTC \\
FvPG-R & TCATCGTTGTCAAGAAGACCA \\
Fv-cellulase-F & GTTCACAATCTGCGAGTTCAAG \\
Fv-cellulase-R & GCTGTAACGAGCTCGACATC \\
& CTGCACCTTGACGATGTTCTT \\
\hline
\end{tabular}


geneticin $\left(250 \mu \mathrm{g} \mathrm{ml}^{-1}\right.$; Research Products International). After 57 days of incubation at $25{ }^{\circ} \mathrm{C}$, drug-resistant colonies were selected for further study.

Nucleic acid manipulations, Southern and Northern blot analyses, and PCR conditions. Bacterial plasmids were isolated with the Wizard miniprep DNA purification system (Promega). Fungal genomic DNA was isolated from the frozen mycelium as previously described (Shim \& Woloshuk, 2001). Total RNA samples were isolated with Trizol reagent (Invitrogen) by following the manufacturer's suggested protocol. Standard molecular biology techniques, including Southern and Northern analyses, were performed as described by Sambrook \& Russell (2001). Restriction enzyme-digested (BamHI or EcoRI) fungal genomic DNA $(7 \mu \mathrm{g})$ was subjected to electrophoresis in a $1.0 \%(\mathrm{w} / \mathrm{v})$ agarose gel and transferred onto a Hybond-N nylon membrane (Amersham) for Southern analysis. For Northern hybridization, total RNA $(15 \mu \mathrm{g})$ was separated by electrophoresis on a $1.2 \%(\mathrm{v} / \mathrm{v})$ formaldehyde agarose gel and blotted onto a Hybond-N nylon membrane (Amersham).

The DNA probes were ${ }^{32} \mathrm{P}$-labelled with a Prime-It II random primer labelling kit (Stratagene). All primers used in this study are listed in Table 1. For probes NT, CT, CC and DS, primer pairs FSR-D2+FSRU1, WD40-FW + FSR-U3, CoilBD-FW + CoilBD-RV and FSR-1186FW+FSR-BD-RV, respectively, were used to amplify the DNA fragments from the $F$. verticillioides genome. F. verticillioides AMY1 (GenBank DQ143884) has been isolated and characterized by Bluhm \& Woloshuk (2005). The sequence of the pectinase (PTN) and cellulase (CEL) genes were obtained from the Fusarium Group Database housed at the Broad Institute of the Massachusetts Institute of Technology (http://www.broad.mit.edu/annotation/genome/fusarium_group/ MultiHome.html) using Gibberella fujikuroi pectinase (GenBank Q017181) and Fusarium oxysporum cellulase (GenBank P46237) as query. To amplify specific probes for $A M Y 1, P T N$ and $C E L$, primer pairs Fv-amylase-F+Fv-amylase-R, FvPG-F + FvPG-R and Fvcellulase-F $+\mathrm{Fv}$-cellulase- $\mathrm{R}$ were used, respectively.

Virulence assay. The stalk rot assays were performed as described previously (Shim et al., 2006). Briefly, 8-week-old B73 maize stalks (near pollinating stage) were wounded with a sterile tooth pick and infected with fungal strains. Agar blocks for inoculation were prepared from wild-type, $\Delta f_{s} r 1, \quad f_{s} 1 \Delta C T 5, \quad f_{s} 1 \Delta N T 2$, fsr $1 \Delta C T \Delta C a v 9$, fsr $1 \Delta C T \Delta C C 3$ and $\Delta a m y 1$ strains grown on V8 agar plates (at $25{ }^{\circ} \mathrm{C}$ for 7 days) with a cork borer $(1 \mathrm{~cm}$ in diameter) and placed on wound sites. Infected maize plants were incubated in a growth chamber with controlled temperature $\left(25^{\circ} \mathrm{C}\right)$, relative humidity $(70 \%)$ and light cycle ( $14 \mathrm{~h} \mathrm{light} / 10 \mathrm{~h}$ dark). Plants were incubated for 10 days, and stalks were split longitudinally to inspect the extent of rot.

Hydrolysis of starch. The ability of all mutants to degrade starch was determined by culturing them on starch agar plates $\left[0.3 \mathrm{M} \mathrm{NaNO}_{3}\right.$, $6 \mathrm{mM} \mathrm{K}_{2} \mathrm{HPO}_{4}, 4 \mathrm{mM} \mathrm{MgSO}, 7 \mathrm{mM} \mathrm{KCl}, 66 \mu \mathrm{M} \mathrm{FeSO}_{4}, 2 \%(\mathrm{w} / \mathrm{v})$ starch, $2 \%(\mathrm{w} / \mathrm{v})$ agar]. After anaerobic incubation at $25{ }^{\circ} \mathrm{C}$ for 3 days, the plates were flooded with an iodine solution $[0.5 \%(\mathrm{v} / \mathrm{v})$ iodine, $5 \%(\mathrm{v} / \mathrm{v})$ potassium iodide], as described by Bluhm \& Woloshuk (2005).

\section{RESULTS}

\section{Constructing FSR1 $\mathbf{N}$ terminal-truncated (fsr1 $\Delta N T)$ and C terminal-truncated (fsr1 $\triangle C T)$ F. verticillioides strains}

Based on in silico analysis of the four putative proteinbinding domains in the Fsrl protein, i.e. a caveolin-binding motif, a coiled-coil structure, and a calmodulin-binding motif at the $\mathrm{N}$ terminus and a WD40 repeat domain at the C terminus (Shim et al., 2006), it was conceivable that any one, or a combination of two or more, of the four motifs could play a key role in F. verticillioides virulence (Fig. 1a). After in silico analysis and literature review (Ganem et al., 2004; Pöggeler \& Kück, 2004; Shim et al., 2006; Smith et al., 1999), we hypothesized that the C terminus of Fsrl, containing the WD40 repeats motif, is directly associated with maize stalk rot virulence. To test our hypothesis, we first complemented the FSR1 knockout strain $\left(\Delta f_{s} r 1\right)$ with mutated versions of the FSR1 gene (Fig. 1b). Two complementation constructs, one with a complete deletion of the N-terminus region ( $f s r 1 \Delta \mathrm{NT})$ and the other with a complete deletion of the C-terminus region ( $\mathrm{fsr} 1 \Delta \mathrm{CT}$ ), were developed by the double-joint PCR strategy ( $\mathrm{Yu}$ et al., 2004) (Fig. 1b). Both constructs contained the native promoter of FSR1 and were fused to a geneticin-resistance gene $(G E N)$ as the selectable marker. The two complementation constructs were independently introduced into $\Delta f s r 1$ protoplasts, and the geneticin-resistant colonies were recovered as described previously (Shim et al., 2006). We selected three fsr1 $\Delta$ NT-complemented transformants ( $f$ sr $1 \Delta N T 1, f s r 1 \Delta N T 2$ and $\left.f_{s r} 1 \Delta N T 5\right)$ and three fsr $1 \Delta \mathrm{CT}$ complemented transformants (fsr $1 \Delta C T 5, f s r 1 \Delta C T 7$ and $f_{s r 1 \Delta C T 10)}$ for further molecular characterization. Southern analysis showed that the NT and CT probes hybridized to the wild-type genomic DNA but did not hybridize to the $\Delta f s r 1$ genomic DNA (Fig. 1c), which confirms that the complementation constructs were properly introduced into the transformants. Notably, fsr $1 \Delta C T 5$ and $f_{s} r 1 \Delta N T 2$ transformants were the only strains that harboured a single copy of fsr $1 \Delta \mathrm{CT}$ and fsr $1 \Delta \mathrm{NT}$ constructs, respectively (Fig. 1c). Subsequently, we performed Northern analyses to verify that the fsr $1 \Delta \mathrm{NT}$ and fsr $1 \Delta \mathrm{CT}$ constructs in the transformants were properly expressed. The anticipated $2.8 \mathrm{~kb}$ transcript was detected in the wild-type strain but not in $\Delta f s r 1$ strain when probed with NT and CT (Fig. 1d). The $f_{s r} 1 \Delta C T 5$ and $f_{s r} 1 \Delta C T 10$ strains revealed a high level of $f_{s r} 1 \Delta C T$ expression when the blot was hybridized with the NT probe (Fig. 1d). A high level of fsr $1 \Delta \mathrm{NT}$ expression was observed in $f_{s} 1 \Delta N T 1$, $f_{s r} 1 \Delta N T 2$ and $f s r 1 \Delta N T 5$ transformants when the blot was hybridized with the CT probe (Fig. 1d). We concluded that fsr $1 \Delta \mathrm{NT}$ and fsr $1 \Delta \mathrm{CT}$ constructs successfully integrated in the $\Delta f s r 1$ genome and were properly expressed, particularly in single-insertion transformants $f_{s} r \Delta C T 5$ and $f_{s r} 1 \Delta N T 2$.

\section{The C-terminal region of Fsr1, which contains a WD40 repeat domain, is dispensable for maize stalk rot virulence}

We observed a difference in growth rate and morphology when the mutants were grown on PDA plates. The colonies of the wild-type strain produced aerial mycelia, whereas the colonies of the $\Delta f s r 1$ strain produced fewer aerial mycelia. The $\Delta f s r 1$ strain also exhibited increased reddish-brown pigmentation, and displayed slower radial growth (Fig. 2a). 

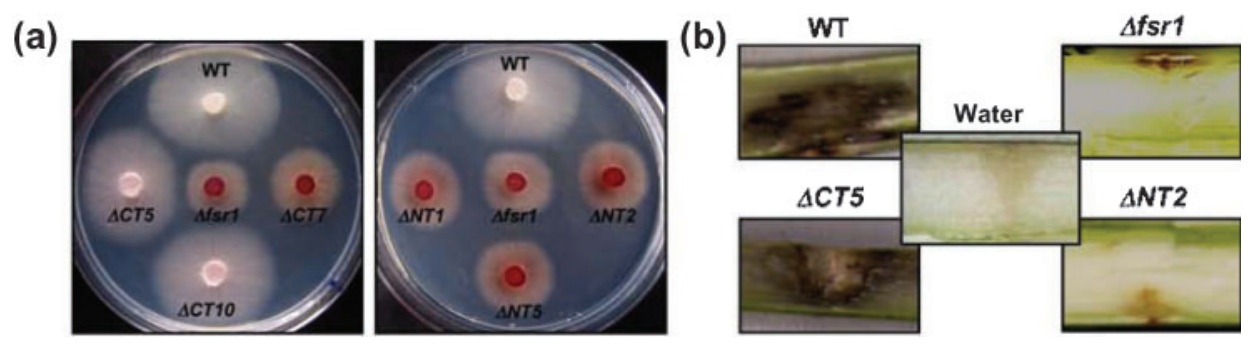

Fig. 2. Growth and virulence assay. (a) Phenotypic comparison of wild-type (WT) and $\triangle f s r 1$ against fsr $1 \Delta C T$ ( $\triangle C T 5, \triangle C T 7$ and $\triangle C T 10$; left panel) and $f s r 1 \Delta N T(\triangle N T 1, \Delta N T 2$ and $\triangle N T 5$; right panel) transformants. All strains were grown on $0.2 \times$ PDA plates for 4 days. (b) Virulence assay performed on B73 corn stalks. Maize stalks inoculated with WT, $\Delta f s r 1, f s r 1 \Delta C T 5$ and fsr $1 \triangle N T 2$, and a water-inoculated negative control, were photographed at 10 days post-inoculation.

Interestingly, growth rates of $f_{s r} 1 \Delta C T 5$ and $f_{s r} 1 \Delta C T 10$ recovered to be similar to that of the wild-type progenitor, which is consistent with the FSR1 gene expression level we observed in the Northern blot (Fig. 1d). Growth in $f_{s} 1 \Delta C T 7$ strain, however, was not restored (Fig. 2a), and this may be due to construct damage during transformation (Fig. 1c). On the other hand, fsr1 $\Delta N T 1$, fsr1 $f_{N T 2}$ and $f_{s} 1 \Delta N T 5$ mutants all mimicked the $\Delta f_{s} r 1$ phenotype with low growth rates and increased reddish-brown pigmentation (Fig. 2a), regardless of the FSR1 gene expression levels (Fig. 1d). Based on molecular and phenotypic characterization, we selected the $f_{s} r \Delta C T 5$ and $f_{s} r 1 \Delta N T 2$ strains for a maize stalk rot assay. Maize plants were inoculated with wild-type, $\Delta f_{s} r 1, \quad f_{s} r 1 \Delta N T 2$ and $f_{s r 1 \Delta C T 5}$ strains as described previously (Shim et al., 2006). After 10 days of incubation, the positive control (wild-type strain) and the negative control $(\Delta f s r 1$ strain) infection assays yielded anticipated rot and no-rot results, respectively (Fig. 2b). The fsr $1 \Delta C T 5$ strain showed vigorous growth and colonization of maize stalks, resulting in severe rot, whereas the fsr1 $\Delta N T 2$ mutant caused little rot and failed to penetrate and grow into maize stalks (Fig. 2b). These results clearly demonstrated that the C-terminal region of the Fsr1 protein, which contains a WD40 repeat domain, is dispensable for virulence, and suggest that a motif(s) critical for regulating maize stalk rot virulence in $F$. verticillioides is present in the $\mathrm{N}$ terminus of the Fsr1 protein.

\section{Impact of Fsr1 on selected CWDEs in $F$. verticillioides}

It is generally acknowledged that many plant-pathogenic fungi can produce a variety of CWDEs that are involved in plant pathogenesis (Lalaoui et al., 2000; Lehtinen, 1993). These enzymes are particularly important for so-called 'rot' pathogens when attacking their hosts by breaking down host tissue (Lehtinen, 1993). Consequently, we examined whether mutations in FSR1 affect the expression of genes that encode CWDEs in $F$. verticillioides. We selected pectinase $(P T N)$ and cellulase (CEL, endo-1,4- $\beta$-glucanase) as the key CWDEs, and investigated the transcript level of these genes in the wild-type and FSR1 mutant strains. We also included $\alpha$-amylase 1 (AMY1), a key enzyme that is responsible for starch metabolism in fungi, in the study. Importantly, Bluhm \& Woloshuk (2005) have shown that $A M Y 1$ is directly associated with fusarium kernel rot and fumonisin $\mathrm{B}_{1}$ production in $F$. verticillioides.

F. verticillioides strains were grown in $10 \%$ crushed maizestalk liquid medium, and fungal mass was harvested for total RNA extraction. Subsequently, we performed Northern analysis to investigate the impact of different FSR1 mutations on the transcript levels of AMY1, PTN and $C E L$. Interestingly, no PTN expression was detected in any strain tested, including the wild-type strain, whereas high CEL expression was observed in all strains (Fig. 3a). Strikingly, we observed a lower $A M Y 1$ expression in $\Delta f_{s} r 1$ and $f_{s} 1 \Delta N T 2$ mutants than in the wild-type strain, suggesting that the $\mathrm{N}$-terminal region of the Fsrl protein is necessary for proper $F$. verticillioides AMY1 gene expression (Fig. 3a). Furthermore, to test whether downregulation of $A M Y 1$ is directly associated with enzyme activity, we performed an amylase activity assay on all four strains. While wild-type and $f s r 1 \Delta C T 5$ strains were able to digest starch, it was clear that $\Delta f s r 1$ and $f s r 1 \Delta N T 2$ were restricted in the ability to utilize starch as the sole carbon source (Fig. 3b). Therefore, we concluded that a complete Fsr1 $\mathrm{N}$ terminus is important for proper $\alpha$-amylase activity in $F$. verticillioides.

From our earlier result, we showed that the $f_{s} r \Delta N T 2$ strain is avirulent when inoculated on maize stalks. We were curious to test whether downregulation of $A M Y 1$ in the $f_{s} 1 \Delta N T 2$ strain (Fig. 3a, b) is directly associated with loss of virulence. To test this hypothesis, we inoculated mature maize stalks with the wild-type and the $\Delta a m y 1$ mutant strain, which is completely inhibited in its ability to digest starch (Bluhm \& Woloshuk, 2005), and observed the rot severity after 1 week of incubation. When maize stalks were split longitudinally, we determined that the $\Delta a m y 1$ mutant was indistinguishable from the wild-type strain in its ability to cause maize stalk rot (Fig. 3c). These in vitro results suggest that FSR1-mediated maize stalk rot virulence in F. verticillioides could be independent of CWDE activities. 


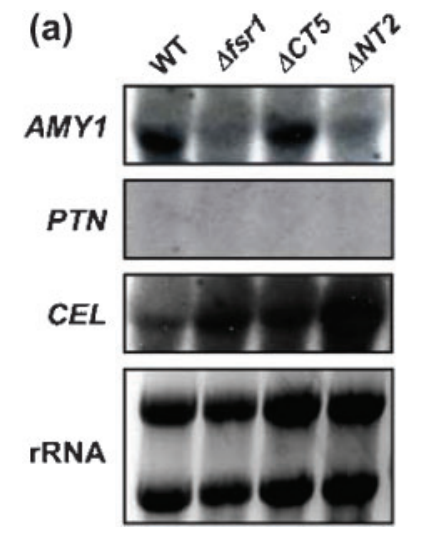

(b)

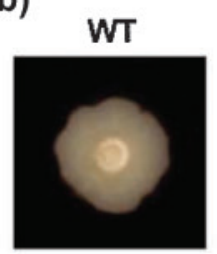

$\triangle C T 5$

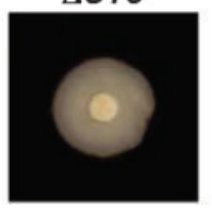

(c)

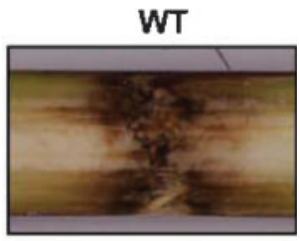

$\triangle N T 2$
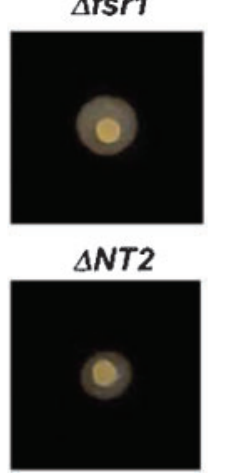

$\Delta a m y 1$

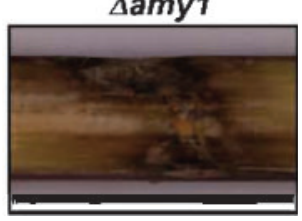

Fig. 3. Analysis of cell wall-degrading enzymes in vitro, produced during infection by $F$. verticillioides mutants. (a) Northern analyses of $\alpha$-amylase 1 ( $A M Y 1$ ), pectinase $(P T N)$ and cellulase (CEL) genes. The wild-type (WT), $\Delta f s r 1, f s r 1 \Delta C T 5$ and fsr1 1 NT2 strains were grown in crude stalk juice broth for 10 days. Total RNA samples (15 $\mu$ g) were subjected to electrophoresis in a $1.2 \%$ denaturing agarose gel. The gel was stained with ethidium bromide to confirm uniformity of loading (rRNA). The ${ }^{32} \mathrm{P}$-labelled $A M Y 1, P T N$ and CEL probes for the Northern analysis were generated via PCR. (b) Production of $\alpha-$ amylase by $F$. verticillioides WT, fsr $1, f s r 1 \Delta C T 5$ and $f s r 1 \Delta N T 2$ strains. Fungi were grown for 3 days on starch agar plates and then stained with an iodine solution; the clear zone around the growth indicates hydrolysis of starch. (c) Virulence assays performed on B73 corn stalks. Maize stalks inoculated with WT and $\Delta a m y 1$ strains were photographed at 10 days postinoculation.

\section{The Fsr1 coiled-coil protein-binding motif is required for maize stalk rot virulence and proper growth in $F$. verticillioides}

Our results suggest that a functional motif(s) present in the $\mathrm{N}$ terminus of the Fsrl protein is essential for virulence (Fig. 2). Of the three potential protein-binding motifs present in the $\mathrm{N}$ terminus, the caveolin-binding domain and coiled-coil structure are highly conserved, whereas the calmodulin-binding motif shares low similarity to other fungal orthologues (Shim et al., 2006). Therefore, we constructed fsr $1 \Delta \mathrm{CT} \Delta \mathrm{Cav}$ and fsr $1 \Delta \mathrm{CT} \Delta \mathrm{CC}$, in which the DNA regions corresponding to the caveolin-binding motif and coiled-coil structure, respectively, were deleted from the fsr $1 \Delta \mathrm{CT}$ construct (Fig. 4a). The $\Delta f_{s} r 1$ protoplasts were transformed with these constructs, and after screening for geneticin-resistant transformants, we selected fsr $1 \Delta C T \Delta \operatorname{Cav} 9$ and $f s r 1 \Delta C T \Delta C C 3$ strains for further characterization.

Southern analysis indicated that $f \operatorname{sr} 1 \Delta C T \Delta \operatorname{Cav} 9$ has a single-copy fsr $1 \Delta \mathrm{CT} \Delta \mathrm{Cav}$ construct in the genome (Fig. $4 \mathrm{~b})$. Furthermore, when the blot was hybridized to the CC probe, a smaller-size transcript $(1.3 \mathrm{~kb})$ was observed in the mutant (Fig. 4a), which is indicative of caveolinbinding motif deletion. Insertion of the fsr $1 \Delta \mathrm{CT} \Delta \mathrm{CC}$ construct into the $\Delta f s r 1$ strain was also confirmed by Southern analysis (Fig. 4b). We subsequently determined by Northern analysis that the truncated gene $(1.2 \mathrm{~kb})$ is expressed in the transformant $f_{s} r \Delta C T \Delta C C 3$ (Fig. 4b). In addition, we also verified via quantitative real-time PCR that no coiled-coil motif expression could be detected in fsr1 $\triangle C T \Delta C C 3$ (data not shown).
The phenotype of the $f_{s} r 1 \Delta C T \Delta \operatorname{Cav} 9$ strain appeared much like that of the wild-type strain in all aspects of growth on PDA (Fig. 4c). When we inoculated the mutant strain on an 8-week-old maize stalk, severe rot symptoms developed after 1 week of incubation (Fig. 4c). In contrast, fsr $1 \triangle C T \Delta C C 3$ grew more slowly on PDA, while producing a higher level of carmine red pigment, similar to $\Delta f s r 1$. Also, virulence was not restored in $f_{s} r 1 \Delta C T \Delta C C 3$ (Fig. 4c). From these observations, we demonstrated that the Frr1 coiled-coil protein-binding motif, not the caveolin-binding domain, is required for maize stalk rot virulence and proper growth in F. verticillioides.

\section{DISCUSSION}

Fsr1 shares high similarity to a unique group of animal proteins, e.g. striatin, zinedin and SG2GA (Shim et al., 2006). The striatin gene is mainly expressed in neurons of the mammalian central nervous system, and the protein contains multiple functional domains involved in protein-protein interactions (Bartoli et al., 1998; Castets et al., 2000; Moreno et al., 2000). It acts as a scaffolding protein, and is involved in transducing and trafficking signals in mammalian systems (Gaillard et al., 2006). Notably, striatin is absent in plants and budding yeast (Benoist et al., 2006); however, there are reports of a striatin orthologue in Drosophila melanogaster (Chen et al., 2002) and the filamentous fungus S. macrospora (Pöggeler \& Kück, 2004). A review of the literature and results from our previous study (Shim et al., 2006) prompted us to further characterize Fsr1, mainly to identify the specific domain(s) in Fsr1 directly associated with F. verticillioides maize stalk rot virulence. 


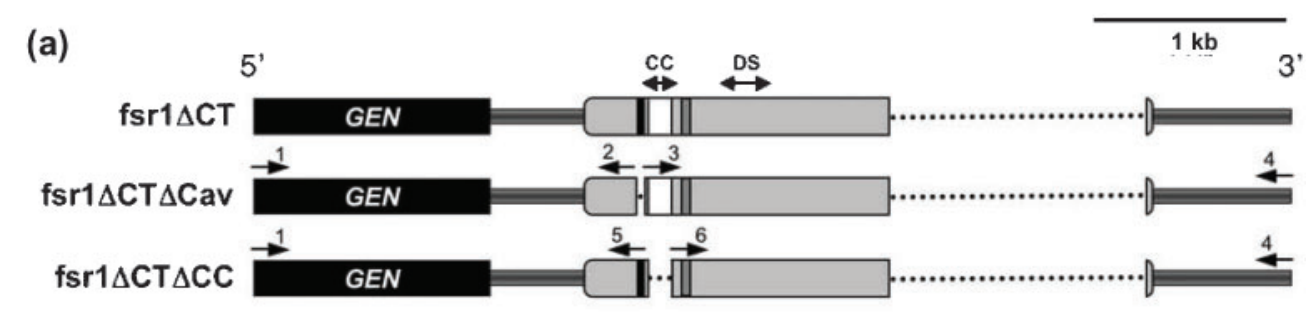

(b)

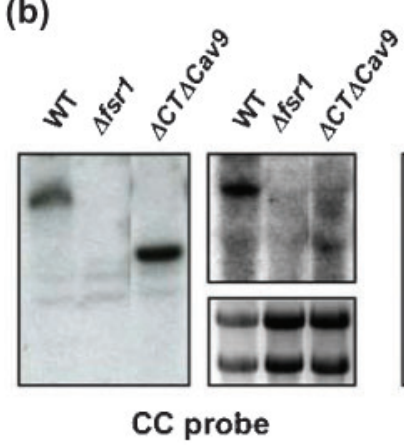

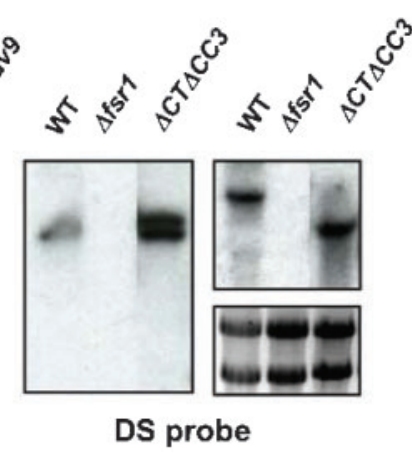

(c)

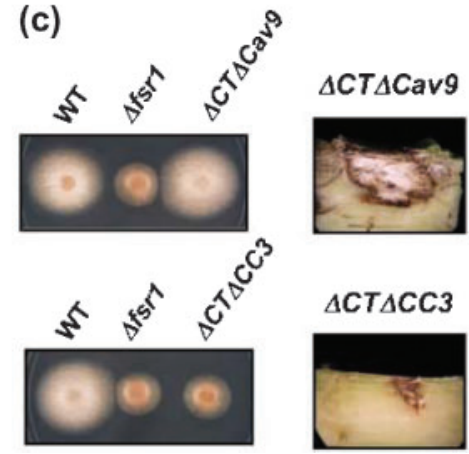

Fig. 4. Functional characterization of Fsr1 $\mathrm{N}$ terminus motifs. (a) Schematic depiction of $\mathrm{N}$-terminal domain complementation constructs. The nucleotide sequences that correspond to the caveolin-binding domain, coiled-coil structure and calmodulinbinding motif are represented as black, white and shaded boxes, respectively. Dotted lines indicates deleted gene regions. The fsr $1 \Delta \mathrm{CT}$ construct was used a template to generate fsr $1 \Delta \mathrm{CT} \Delta \mathrm{Cav}$ and fsr $1 \Delta \mathrm{CT} \Delta \mathrm{CC}$ constructs. Black arrows (CC and DS) show the probe sites for Southern and Northern blots. GEN, geneticin resistance. Arrows depict the primers used to amplify the specific DNA fragments: $1=\mathrm{M} 13 \mathrm{~F}, 2=\mathrm{h}-\mathrm{RV}, 3=\mathrm{g}-\mathrm{FW}, 4=\mathrm{FVLM} 2 \mathrm{~F} 1,5=\mathrm{j}-\mathrm{RV}$, and $6=\mathrm{i}-\mathrm{FW}$. (b) Southern (left panel) and Northern (right panel) blot analysis of fsr $1 \Delta C T \Delta C a v 9$ and $f s r 1 \triangle C T \Delta C C 3$. Fungal genomic DNA samples were digested with EcoRI and subjected to electrophoresis in $1 \%$ agarose gel. Total RNA samples $(15 \mu \mathrm{g})$ were subjected to electrophoresis in a $1.2 \%$ denaturing agarose gel. The gel was stained with ethidium bromide to confirm uniformity of loading (rRNA; bottom-right panels). The blots were hybridized with ${ }^{32} \mathrm{P}$-labelled CC and DS probes (shown in Fig. 4a), respectively. (c) Growth and virulence phenotypes of $f s r 1 \Delta C T \Delta C a v$ and $f s r 1 \Delta C T \Delta C C 3$ strains. Strains were grown on a $0.2 \times$ PDA plate, and the growth pattern was observed after 4 days of incubation. The virulence assay was performed on B73 corn stalks. The maize stalk rot assay of $f_{s} 1 \triangle C T \Delta C C 3$ was photographed at 10 days post-inoculation.

We examined the role of individual domains in fungal virulence by generating a series of motif-deletion mutations in FSR1 ( $f$ sr1 $1 \Delta N T, f s r 1 \Delta C T, f s r 1 \Delta C T \Delta C a v$ and fsr $1 \Delta C T \Delta C C$ ) (Figs $1 \mathrm{~b}$ and $4 \mathrm{a}$ ). Our initial hypothesis was that the WD40 repeat domain in Fsr1 is essential for stalk rot virulence. The WD40 repeats are found in a number of eukaryotic proteins involved in signal transduction, RNA processing, gene regulation, cell division, cytoskeleton assembly and protein degradation (Smith et al., 1999). Typically, the WD repeat is a 40 to 60 -residue sequence that contains a glycine-histidine $(\mathrm{GH})$ dipeptide 11-24 residues from its $\mathrm{N}$ terminus and a tryptophanaspartate (WD) dipeptide at its $\mathrm{C}$ terminus. This domain is predicted to form a propeller-like structure, creating a stable platform that enables the protein to coordinate interactions with other proteins and/or small ligands (Smith et al., 1999). The best-characterized WD-repeat protein is the $\mathrm{G} \beta$ subunit of heterotrimeric $G$ protein, which is a well-characterized component of eukaryotic transmembrane signalling pathways (Smith et al., 1999). The Fsr1 WD40 repeat motif shares $74 \%$ amino acid identity with the corresponding motif in S. macrospora
Pro11, and the key amino acids are highly conserved (Shim et al., 2006). To test our hypothesis, we first divided the Fsr1 protein into two larger regions, the $\mathrm{N}$-terminus and C-terminus regions, instead of individually targeting all four putative motifs. Strikingly, contrary to our expectation, the $f_{s} 1 \Delta C T 5$ mutant fully recovered virulence when inoculated on maize stalks (Fig. 2b), whereas the fsr $1 \Delta N T 2$ mutants showed near complete loss of pathogenicity on maize plants (Fig. 2b). Thus, we formulated an alternative hypothesis that a functional motif(s) present in the $\mathrm{N}$ terminus of the Fsr1 protein holds the key to maize stalk rot virulence.

Subsequent experiments determined that the coiled-coil motif in the Fsr1 $\mathrm{N}$ terminus is crucial for F. verticillioides virulence (Fig. 4c). In addition to loss of virulence, deletion of the coiled-coil domain ( $\left.f_{s} r 1 \Delta C T \Delta C C 3\right)$ causes multiple aberrant phenotypes that include elevated red-pigment production and slower growth, suggesting that the coiledcoil domain may affect multiple downstream genes. Coiled-coil motifs are known to be responsible for many protein-protein interactions (Newman et al., 2000), 
suggesting an important functional role in signal transduction. Patharkar \& Cushman (2006) have reported that ice plant (Mesembryanthemum crystallinum) McCap1, a coiled-coil protein, interacts with McCpk1 (M. crystallinum calcium-dependent protein kinase 1) and is responsible for changes in its localization from the plasma membrane to the nucleus following exposure to low humidity. In particular, regulation of virulence via protein-protein interactions is frequently observed in eukaryotic systems, including fungi. For example, Fbp 1 (F-box protein 1) in $F$. graminearum participates in the formation of the $\mathrm{SCF}^{\mathrm{FBP} 1}$ complex that controls the ubiquitin-mediated degradation of proteins involved in sexual reproduction and virulence (Han et al., 2007). Cui et al. (2002) have generated a variety of spontaneous mutations in Botryotinia fuckeliana that have resulted in dicarboximide-fungicide resistance and osmotic-sensitivity phenotypes. The resistant strains show a variety of single amino-acid differences in the putative coiled-coil domain in the BOS1 gene (encoding osmosensing histidine kinase), suggesting that the fungicide may specifically interact with this region (Cui et al., 2002). In addition to mediating function through protein-protein interactions, Gaillard et al. (2006) have shown that the coiled-coil domain in the striatin protein determines the localization of the protein in spines and controls the oligomerization of members of the striatin family. Our study demonstrates that the coiled-coil motif is a key regulatory domain that regulates fungal virulence in $F$. verticillioides, and opens up a new avenue to investigate the mechanism of fungal virulence signalling. With an understanding that the coiled-coil domain is an important motif in protein-protein interactions in vivo and that this interaction may perhaps trigger downstream gene signalling associated with $F$. verticillioides virulence, our future studies will aim to identify and characterize putative Fsr1 coiled-coil motif binding proteins.

Another interesting observation from this study was the perspective that the FSR1-mediated virulence signalling pathway in F. verticillioides may not be directly associated with production of CWDEs. Many plant-pathogenic fungi produce various CWDEs for successful infection of host plants. In particular, endo-polygalacturonase (pectinase) is considered one of the key CWDEs associated with plant pathogenesis. Pectinases in Erwinia carotovora (Lei et al., 1985) and Claviceps purpurea (Oeser et al., 2002) are essential for virulence. Furthermore, at least five pectinase genes are present in Botrytis cinerea (ten Have et al., 1998) and F. oxysporum (Di Pietro \& Roncero, 1998; GarciaMaceira et al., 2001), and have been shown to have functional roles in fungal virulence and host infection. However, it is interesting to note that in some fungal species, e.g. Cochliobolus carbonum, pectinase is not directly required for pathogenesis (Scott-Craig et al., 1990). Cellulase, however, is unlikely to be an important enzyme directly associated with host attack, because extensive cellulose degradation typically occurs only late in infection, suggesting that it may not be the determining factor for pathogen aggressiveness in the early stages of pathogenesis (Novo et al., 2006; Walton, 1994). On the other hand, cellulases of phytopathogenic bacteria may be directly responsible for pathogen aggressiveness, since cellulase gene disruption in Pseudomonas solanacearum, Xanthomonas campestris pv campestris, E. carotovora and Erwinia chrysanthemi all result in loss of virulence (BortoliGerman et al., 1994; Gough et al., 1988; Mae et al., 1995; Roberts et al., 1988). Interestingly, our Northern blotting data suggested a possible link between FSR1 and the $\alpha$ amylase gene, but not between the selected cellulase and pectinase genes, during maize stalk rot development (Fig. 3a). $\alpha$-Amylase is a key starch-utilizing enzyme in fungi, and in F. verticillioides the enzyme has been shown to play an important role in maize kernel colonization and concomitant production of the mycotoxin fumonisin $B_{1}$ (Bluhm \& Woloshuk, 2005). Significantly, when we performed a maize stalk rot assay with the $\Delta a m y 1$ mutant strain, we observed no difference in rot virulence when compared to the wild-type progenitor (Fig. 3c), demonstrating that reduced amylase activity does not negatively impact $F$. verticillioides maize stalk rot virulence. However, it is important to note that additional genes that encode CWDEs are present in the F. verticillioides genome. A Fusarium Group Database search revealed eight putative amylase genes, seven putative pectinase genes and three putative cellulase genes, and therefore we cannot rule out the possibility that FSR1 controls other CWDE genes during stalk rot pathogenesis. Further in planta experiments are necessary to unambiguously resolve this hostpathogen interaction mechanism.

\section{ACKNOWLEDGEMENTS}

We thank Dr Charles P. Woloshuk (Purdue University, West Lafayette, IN) for providing the $\Delta a m y 1$ strain of $F$. verticillioides. We also thank Drs Brian Shaw and Clint Magill for critical reading of this manuscript. This work was supported by the National Research Initiative of the United States Department of Agriculture (USDA) Cooperative State Research, Education and Extension Service (grant no. 2007-35319-18334).

\section{REFERENCES}

Bartoli, M., Monneron, A. \& Ladant, D. (1998). Interaction of calmodulin with striatin, a WD-repeat protein present in neuronal dendritic spines. J Biol Chem 273, 22248-22253.

Benoist, M., Gaillard, S. \& Castets, F. (2006). The striatin family: a new signaling platform in dendritic spines. J Physiol (Paris) 99, 146153.

Bluhm, B. H. \& Woloshuk, C. P. (2005). Amylopectin induces fumonisin B1 production by Fusarium verticillioides during colonization of maize kernels. Mol Plant Microbe Interact 18, 13331339.

Bortoli-German, I., Brun, E., Py, B., Chippaux, M. \& Barras, F. (1994). Periplasmic disulphide bond formation is essential for cellulase secretion by the plant pathogen Erwinia chrysanthemi. Mol Microbiol 11, 545-553. 
Castets, F., Bartoli, M., Barnier, J. V., Baillat, G., Salin, P., Moqrich, A., Bourgeois, J. P., Denizot, F., Rougon, G. \& other authors (1996). A novel calmodulin-binding protein, belonging to the WD-repeat family, is localized in dendrites of a subset of CNS neurons. J Cell Biol 134, 1051-1062.

Castets, F., Rakitina, T., Gaillard, S., Moqrich, A., Mattei, M. G. \& Monneron, A. (2000). Zinedin, SG2NA, and striatin are calmodulinbinding, WD repeat proteins principally expressed in the brain. J Biol Chem 275, 19970-19977.

Chen, H. W., Marinissen, M. J., Oh, S. W., Chen, X., Melnick, M., Perrimon, N., Gutkind, J. S. \& Hou, S. X. (2002). CKA, a novel multidomain protein, regulates the JUN N-terminal kinase signal transduction pathway in Drosophila. Mol Cell Biol 22, 1792-1803.

Cui, W., Beever, R. E., Parkes, S. L., Weeds, P. L. \& Templeton, M. D. (2002). An osmosensing histidine kinase mediates dicarboximide fungicide resistance in Botryotinia fuckeliana (Botrytis cinerea). Fungal Genet Biol 36, 187-198.

Desjardins, A. E., Munkvold, G. P., Plattner, R. D. \& Proctor, R. H. (2002). FUM1 - a gene required for fumonisin biosynthesis but not for maize ear rot and ear infection by Gibberella moniliformis in field tests. Mol Plant Microbe Interact 15, 1157-1164.

Di Pietro, A. \& Roncero, M. I. (1998). Cloning, expression, and role in pathogenicity of $p g 1$ encoding the major extracellular endopolygalacturonase of the vascular wilt pathogen Fusarium oxysporum. Mol Plant Microbe Interact 11, 91-98.

Flaherty, J. E., Pirttila, A. M., Bluhm, B. H. \& Woloshuk, C. P. (2003). $P A C 1$, a pH-regulatory gene from Fusarium verticillioides. Appl Environ Microbiol 69, 5222-5227.

Gaillard, S., Bailly, Y., Benoist, M., Rakitina, T., Kessler, J. P., Fronzaroli-Molinieres, L., Dargent, B. \& Castets, F. (2006). Targeting of proteins of the striatin family to dendritic spines: role of the coiledcoil domain. Traffic 7, 74-84.

Ganem, S., Lu, S. W., Lee, B. N., Chou, D. Y. T., Hadar, R., Turgeon, B. G. \& Horwitz, B. A. (2004). G-protein beta subunit of Cochliobolus heterostrophus involved in virulence, asexual and sexual reproductive ability, and morphogenesis. Eukaryot Cell 3, 1653-1663.

Garcia-Maceira, F. I., Di Pietro, A., Huertas-Gonzalez, M. D., Ruiz-Roldan, M. C. \& Roncero, M. I. (2001). Molecular characterization of an endopolygalacturonase from Fusarium oxysporum expressed during early stages of infection. Appl Environ Microbiol 67, 2191-2196.

Gough, C. L., Dow, J. M., Barber, C. E. \& Daniels, M. J. (1988). Cloning of two endoglucanase genes of Xanthomonas campestris pv campestris: analysis of the role of the major endoglucanase in pathogenesis. Mol Plant Microbe Interact 1, 275-281.

Han, Y. K., Kim, M. D., Lee, S. H., Yun, S. H. \& Lee, Y. W. (2007). A novel F-box protein involved in sexual development and pathogenesis in Gibberella zeae. Mol Microbiol 63, 768-779.

Lalaoui, F., Halama, P., Dumortier, V. \& Paul, B. (2000). Cell walldegrading enzymes produced in vitro by isolates of Phaeosphaeria nodorum differing in aggressiveness. Plant Pathol 49, 727-733.

Lehtinen, U. (1993). Plant cell wall degrading enzymes of Septoria nodorum. Physiol Mol Plant Pathol 43, 121-134.

Lei, S. P., Lin, H. C., Heffernan, L. \& Wilcox, G. (1985). Evidence that polygalacturonase is a virulence determinant in Erwinia carotovora. J Bacteriol 164, 831-835.
Mae, A., Heikinheimo, R. \& Palva, E. T. (1995). Structure and regulation of the Erwinia carotovora subspecies carotovora SCC3193 cellulase gene celV1 and the role of cellulase in phytopathogenicity. Mol Gen Genet 247, 17-26.

Moreno, C. S., Park, S., Nelson, K., Ashby, D., Hubalek, F., Lane, W. S. \& Pallas, D. C. (2000). WD40 repeat proteins striatin and $S / G_{2}$ nuclear autoantigen are members of a novel family of calmodulin-binding proteins that associate with protein phosphatase 2A. J Biol Chem 275, 5257-5263.

Newman, J. R., Wolf, E. \& Kim, P. S. (2000). A computationally directed screen identifying interacting coiled coils from Saccharomyces cerevisiae. Proc Natl Acad Sci U S A 97, 13203-13208.

Novo, M., Pomar, F., Gayoso, C. \& Merino, F. (2006). Cellulase activity in isolates of Verticillium dahliae differing in aggressiveness. Plant Dis 90, 155-160.

Oeser, B., Heidrich, P. M., Muller, U., Tudzynski, P. \& Tenberge, K. B. (2002). Polygalacturonase is a pathogenicity factor in the Claviceps purpurea/rye interaction. Fungal Genet Biol 36, 176-186.

Patharkar, O. R. \& Cushman, J. C. (2006). A novel coiled-coil protein co-localizes and interacts with a calcium-dependent protein kinase in the common ice plant during low-humidity stress. Planta 225, 57-73.

Pöggeler, S. \& Kück, U. (2004). A WD40 repeat protein regulates fungal cell differentiation and can be replaced functionally by the mammalian homologue striatin. Eukaryot Cell 3, 232-240.

Roberts, D. P., Denny, T. P. \& Schell, M. A. (1988). Cloning of the egl gene of Pseudomonas solanacearum and analysis of its role in phytopathogenicity. J Bacteriol 170, 1445-1451.

Sambrook, J. \& Russell, D. W. (2001). Molecular Cloning: a Laboratory Manual, 3rd edn. Cold Spring Harbor, NY: Cold Spring Harbor Laboratory.

Scott-Craig, J. S., Panaccione, D. G., Cervone, F. \& Walton, J. D. (1990). Endopolygalacturonase is not required for pathogenicity of Cochliobolus carbonum on maize. Plant Cell 2, 1191-1200.

Shim, W. B. \& Woloshuk, C. P. (2001). Regulation of fumonisin $B_{1}$ biosynthesis and conidiation in Fusarium verticillioides by a cyclin-like (C-type) gene, FCC1. Appl Environ Microbiol 67, 1607-1612.

Shim, W. B., Sagaram, U. S., Choi, Y. E., So, J., Wilkinson, H. H. \& Lee, Y. W. (2006). FSR1 is essential for virulence and female fertility in Fusarium verticillioides and F. graminearum. Mol Plant Microbe Interact 19, 725-733.

Smith, T. F., Gaitatzes, C., Saxena, K. \& Neer, E. J. (1999). The WD repeat: a common architecture for diverse functions. Trends Biochem Sci 24, 181-185.

ten Have, A., Mulder, W., Visser, J. \& van Kan, J. A. (1998). The endopolygalacturonase gene Bcpg1 is required for full virulence of Botrytis cinerea. Mol Plant Microbe Interact 11, 1009-1016.

Walton, J. D. (1994). Deconstructing the cell wall. Plant Physiol 104, 1113-1118.

White, D. G. (1999). Compendium of Corn Diseases, 3rd edn. St. Paul, MN: APS Press.

Yu, J. H., Hamari, Z., Han, K. H., Seo, J. A., Reyes-Dominguez, Y. \& Scazzocchio, C. (2004). Double-joint PCR: a PCR-based molecular tool for gene manipulations in filamentous fungi. Fungal Genet Biol 41, 973-981.

Edited by: J.-R. Xu 